\title{
Article
}

\section{Rotational and pulsational variability in the TESS light curve of HD 27463}

Khalack, V, Lovekin, C, Bowman, D M, Kobzar, O, David-Uraz, A, Paunzen, E, Sikora, J, Lenz, P, Kochukhov, O, Holdsworth, Daniel Luke and Wade, G A

Available at http://clok.uclan.ac.uk/30389/

Khalack, V, Lovekin, C, Bowman, D M, Kobzar, O, David-Uraz, A, Paunzen, E, Sikora, J, Lenz, P, Kochukhov, $O$ et al (2019) Rotational and pulsational variability in the TESS light curve of HD 27463. Monthly Notices of the Royal Astronomical Society, 490 (2). pp. 2102-2111. ISSN 0035-8711

It is advisable to refer to the publisher's version if you intend to cite from the work. http://dx.doi.org/10.1093/mnras/stz2782

For more information about UCLan's research in this area go to http://www.uclan.ac.uk/researchgroups/ and search for < name of research Group>.

For information about Research generally at UCLan please go to http://www.uclan.ac.uk/research/

All outputs in CLoK are protected by Intellectual Property Rights law, including Copyright law. Copyright, IPR and Moral Rights for the works on this site are retained by the individual authors and/or other copyright owners. Terms and conditions for use of this material are defined in the policies page.

\section{CLoK}

Central Lancashire online Knowledge www.clok.uclan.ac.uk

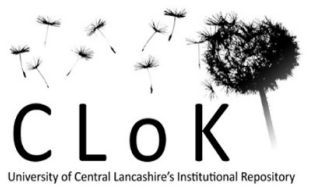




\title{
Rotational and pulsational variability in the TESS light curve of HD 27463
}

\author{
V. Khalack, ${ }^{1 \star}$ C. Lovekin, ${ }^{2}$ D. M. Bowman, ${ }^{3}$ O. Kobzar, ${ }^{1}$ A. David-Uraz ${ }^{\oplus},{ }^{4}$ \\ E. Paunzen ${ }^{\oplus}, 5$ J. Sikora,${ }^{6,7}$ P. Lenz, ${ }^{8}$ O. Kochukhov, ${ }^{9}$ D. L. Holdsworth ${ }^{\oplus 10}$ \\ and G. A. Wade ${ }^{7}$ \\ ${ }^{1}$ Département de Physique et d'Astronomie, Université de Moncton, Moncton, NB, Canada E1A $3 E 9$ \\ ${ }^{2}$ Department of Physics, Mount Allison University, Sackville, NB, Canada E4L 1E6 \\ ${ }^{3}$ Institute of Astronomy, KU Leuven, Celestijnenlaan 200D, 3001 Leuven, Belgium \\ ${ }^{4}$ Department of Physics \& Astronomy, University of Delaware, 217 Sharp Lab, Newark, DE 19716, USA \\ ${ }^{5}$ Department of Theoretical Physics and Astrophysics, Masaryk University, Kotlářská 2, 61137 Brno, Czech Republic \\ ${ }^{6}$ Department of Physics, Engineering Physics \& Astronomy, Queen's University, Kingston, ON, Canada K7L $3 N 6$ \\ ${ }^{7}$ Department of Physics and Space Physics, Royal Military College of Canada, PO Box 17000, Kingston, ON, Canada K7K $7 B 4$ \\ ${ }^{8}$ Ronin Institute, Montclair, NJ 07043, USA \\ ${ }^{9}$ Department of Physics and Astronomy, Uppsala University, Box 516, 75120 Uppsala, Sweden \\ ${ }^{10}$ Jeremiah Horrocks Institute, University of Central Lancashire, Preston PR1 2HE, UK
}

Accepted 2019 October 1. Received 2019 October 1; in original form 2019 June 11

\begin{abstract}
The new photometric data on pulsating Ap star HD 27463 obtained recently with the Transiting Exoplanet Survey Satellite (TESS) are analysed to search for variability. Our analysis shows that HD 27463 exhibits two types of photometric variability. The low-frequency variability with the period $P=2.834274 \pm 0.000008 \mathrm{~d}$ can be explained in terms of axial stellar rotation assuming the oblique magnetic rotator model and presence of surface abundance/brightness spots, while the detected high-frequency variations are characteristics of $\delta$ Scuti pulsations. From the analysis of Balmer line profiles visible in two FEROS spectra of HD 27463 we have derived its effective temperature and surface gravity, finding values that are close to those published for this star in the TESS Input Catalogue (TIC). Knowing the rotation period and the $v \sin i$ value estimated from the fitting of Balmer line profiles we found that the rotational axis is inclined to the line of sight with an angle of $i=33 \pm 8 \mathrm{deg}$. Our best-fitting model of the observed pulsation modes results in an overshoot parameter value $f_{\text {ov }}=0.014$ and values of global stellar parameters that are in good agreement with the data reported in the TIC and with the data derived from fitting Balmer line profiles. This model indicates an age of 5.0 $\pm 0.4 \times 10^{8} \mathrm{yr}$, which corresponds to a core hydrogen fraction of 0.33 .
\end{abstract}

Key words: stars: magnetic field-stars: rotation-stars: oscillations-stars: fundamental parameters -stars: chemically peculiar-stars: individual: HD 27463.

\section{INTRODUCTION}

Ever since a strong peculiarity was discovered in the Si II doublet of the archetypal star $\alpha^{2}$ CVn (Maury \& Pickering 1897), chemically peculiar stars have been the subject of numerous studies. In particular, Ap stars (classified as ' $\mathrm{CP} 2$ ' in contrast to Am, $\mathrm{HgMn}$ or He stars) are known to exhibit periodic brightness variations generally understood to be associated with rotation taking into account that in these stars, strong magnetic fields (Babcock 1958) lead to the creation of surface abundance patches.
A subset of Ap stars lies within the $\delta$ Scuti instability strip. Accordingly, high-frequency pulsations have been discovered in a number of Ap stars, starting with Przybylski's star (HD 101065; Kurtz 1978). Dedicated surveys have revealed a few rapidly oscillating Ap (or 'roAp') stars, but were limited to relatively highamplitude pulsations since they were carried out using ground-based observations. However, ground-based surveys and the advent of high-precision spaced-based photometry have enabled the discovery and detailed study of new Ap pulsators (e.g. HD 24355; Holdsworth et al. 2014, 2016) and the revisiting of known pulsators (e.g. HD 137949; Kurtz 1982; Holdsworth et al. 2018).

The Transiting Exoplanet Survey Satellite (TESS) was launched by NASA on 2018 April 18 with the purpose of detecting exoplanets using the transit method (Ricker et al. 2015). Over the course of its 
2-yr nominal mission, it will cover most of the sky in 26 sectors with its four wide-field cameras, each covering $24 \times 24 \mathrm{deg}$. Each sector is observed for $\sim 27 \mathrm{~d}$, and sectors in each ecliptic hemisphere overlap near the poles. Therefore stars observed by TESS will have temporal baselines between $27 \mathrm{~d}$ and about a year. All fields are observed with a 30-min cadence (in full-frame images, or FFIs) but a subset (>200 000) of stars has been selected for short-cadence (2min) observations, forming the candidate target list (CTL; Stassun et al. 2018, 2019). With such a temporal baseline and cadence, TESS not only represents a formidable asset for exoplanetary detection, but can also be leveraged very profitably for asteroseismology (e.g. Campante et al. 2016). As such, this mission represents a tremendous opportunity to not only significantly increase the sample size of known pulsating Ap stars (see e.g. Cunha et al. 2019), but also, given the exquisite data quality, to perform a detailed characterization of specific objects of interest.

The chemically peculiar (CP) star HD 27463 (TT Ret, HIP 19917, Renson 7050, TIC 38586082) is known as an $\alpha^{2}$ CVn type variable with spectral type $\mathrm{Ap} \mathrm{EuCr}(\mathrm{Sr})$ (Houk \& Cowley 1975). Photometric variability of this star with a period of $P=2.835 \mathrm{~d}$ has been reported in the Hipparcos and Tycho catalogues (ESA 1997) and confirmed by Renson \& Catalano (2001).

HD 27463 is a long period $(\sim 370$ yr $)$ visual binary with a separation of $\sim 0.3$ arcsec and a magnitude difference between the Ap primary and the secondary of about 0.43 in the $V$ band (e.g. Baize \& Petit 1989 and references therein) and 0.9 in the Strömgren $y$ band (Hartkopf et al. 2012). Taking into account the observed small angular separation between the two components, they both contribute to the flux detected by TESS.

Previous surveys designed to detect high-frequency pulsations did not yield any detection in this star (Martinez \& Kurtz 1994; Joshi et al. 2016). However, using TESS data, Cunha et al. (2019) and Sikora et al. (2019) have classified HD 27463 as a suspected new $\delta$ Scuti variable. Therefore, in this study, we set out to perform a more involved investigation of this star's TESS photometry, in conjunction with high-resolution spectroscopy. This study is carried out as part of the MOBSTER project, an analysis of magnetic O, B and A stars using TESS light curves (David-Uraz et al. 2019), and of the VeSElkA project, a search for CP stars with vertical stratification of elemental abundance (Khalack \& LeBlanc 2015a,b).

The photometric and spectral observations and the respective reduction procedures are described in Section 2. The use of automatic software to analyse the light curves is considered in Section 3. Comparisons of the fundamental stellar parameters derived from the analysis of Balmer line profiles and from the simulation of stellar pulsations are shown in Section 4. The discussion follows in Section 5.

\section{OBSERVATIONS AND DATA REDUCTION}

HD 27463 has been observed by TESS in Sectors 1-5 in the frame of the TESS guest programme Rotationally-Induced Variability Of Chemically Peculiar (CP) Stars (Ricker \& Vanderspek 2018). The TESS data products have been reduced employing the SPOC pipeline (Jenkins et al. 2016), which is based on the Kepler Science Processing Pipeline. The light curves and the associated data listed in the TESS Input Catalogue (TIC ${ }^{1}$ ) have been downloaded via the Mikulski Archive for Space Telescopes $\left(\mathrm{MAST}^{2}\right.$ ) and are publicly
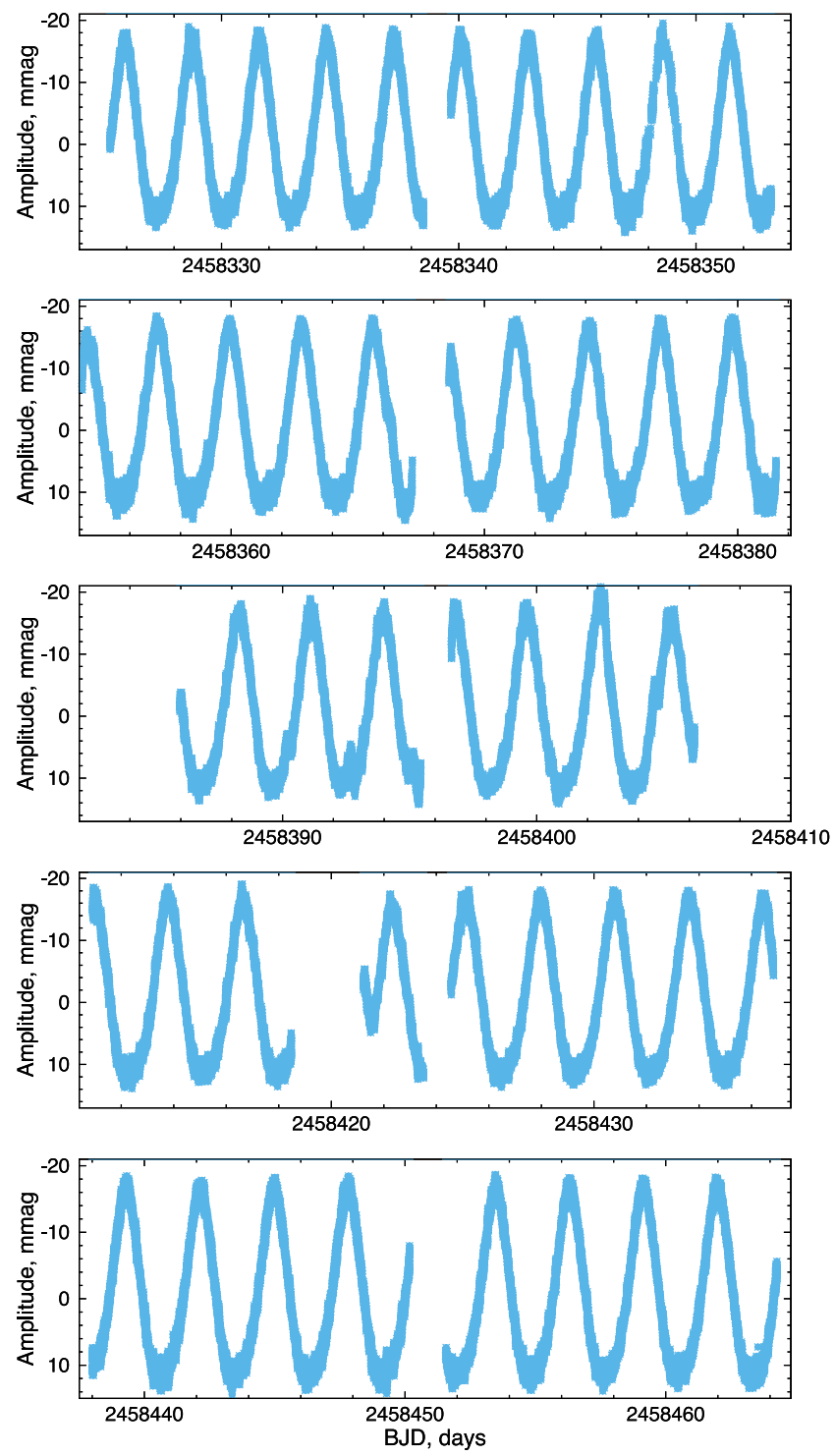

Figure 1. Examples of the detrended light curves obtained by TESS for HD 27463 in Sectors 1-5 (from top to bottom).

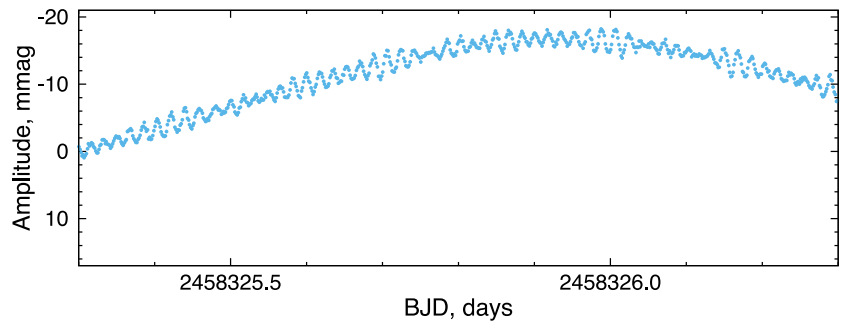

Figure 2. Part of the light curve obtained with TESS for HD 27463 over a time span of $1 \mathrm{~d}$ in Sector 1 . The observed long-term variability is related to the stellar rotation (see Fig. 1). Stellar pulsations with frequencies expected from a $\delta$ Scuti variable and beating of close pulsation frequencies are clearly visible (see also the lower panel in Fig. 3).

\footnotetext{
${ }^{1}$ https://archive.stsci.edu/tess/tic_ctl.html

${ }^{2} \mathrm{http}: / /$ archive.stsci.edu/tess/all products.html
} 

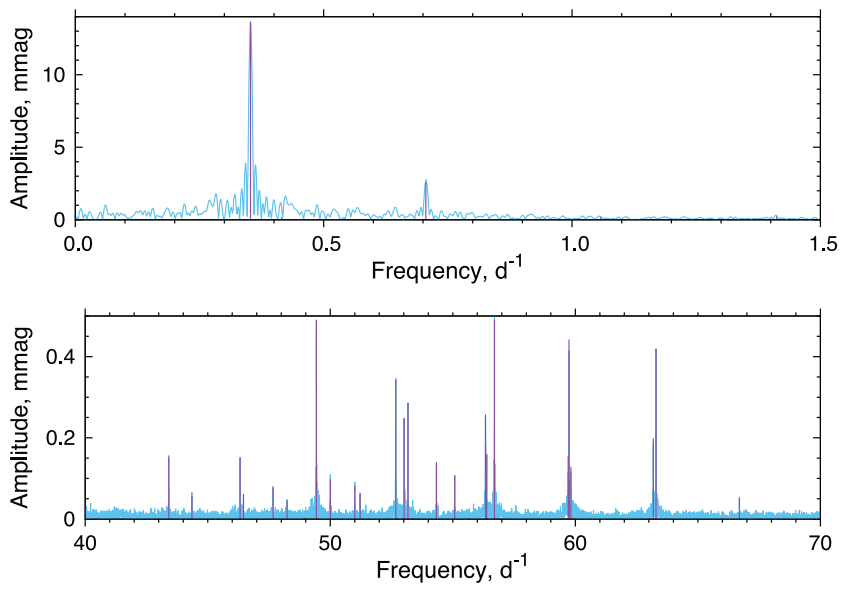

Figure 3. Examples of amplitude spectra derived from the analysis of HD 27463's light curve at low (upper panel) and high (bottom panel) frequencies. Variability at low frequencies is related to rotation of the star, while signals detected at high frequencies are due to stellar pulsations. Vertical lines mark the derived frequencies and their amplitudes for the detected periodic signals (see Table 1)

available. The extracted time series of flux measurements taken at different barycentric Julian dates (BJD) were transformed to units of stellar magnitude. The light curves have been normalized to a mean of zero (see Figs 1, 2).

To accurately determine the rotation period and the pulsation mode frequencies of HD 27463, we further detrended the 2min TESS light curve following the methodology of Bowman et al. (2018). We fitted a periodic rotational modulation model comprising multiple harmonics of the rotation frequency, $v_{\text {rot }}=$ $0.352824 \pm 0.000001 \mathrm{~d}^{-1}$, to the light curve using non-linear least-squares. We subsequently subtracted this preliminary fit and modelled the residuals using a locally weighted scatterplot smoothing (LoWeSS) filter (Cleveland 1979; Seabold \& Perktold 2010). To create our final detrended light curve, we subtracted the modelled residuals from the original light curve. This ensures that a highquality light curve is used for the determination of a rotation period and for the extraction of pulsation mode frequencies, since the longterm trends and instrumental systematics have been removed.

HD 27463 was observed twice in 2008 with the FEROS spectrograph installed on the 2.2-m Max Planck Gesellschaft/European Southern Observatory (MPG/ESO) telescope in La Silla. The FEROS spectrograph provides high-resolution spectra with $R \sim$ 48000 in the spectral region from 3600 to $9200 \AA$ (Kaufer et al. 1999). Two available spectra of HD 27463 were reduced with the ESO automatic reduction pipeline employing the barycentric velocity correction. The spectra are public and have been downloaded from the ESO archive. ${ }^{3}$ To carry out analysis of Balmer line profiles we have used non-normalized spectra (see Section 4) and therefore no additional reduction procedure has been applied to the downloaded data.

\section{PHOTOMETRIC ANALYSIS}

To carry out the analysis of the TESS light curve we have developed automatic software TESS-AP (several scripts and codes including the code PERIOD04) that treats time series, performs periodic analysis,

${ }^{3}$ http://archive.eso.org/wdb/wdb/adp/phase3_spectral/query
Table 1. Characteristics of the periodic signals detected in the light curve of HD 27463 combined from the photometric measurements in the TESS sectors $1-5$.

\begin{tabular}{|c|c|c|c|c|}
\hline $\begin{array}{l}\text { Frequency } \\
\mathrm{d}^{-1}\end{array}$ & $\begin{array}{l}\text { Amplitude } \\
\text { mmag }\end{array}$ & $\begin{array}{l}\text { Phase } \\
\mathrm{rad} / 2 \pi\end{array}$ & $\mathrm{S} / \mathrm{N}$ & $\begin{array}{c}\text { Modes } \\
n\end{array}$ \\
\hline$v_{\text {rot }} 0.352824 \pm 0.000001$ & $13.596 \pm 0.006$ & $0.9359 \pm 0.0001$ & 29.1 & \\
\hline $2 v_{r o t} 0.705639 \pm 0.000006$ & $2.573 \pm 0.007$ & $0.0895 \pm 0.0004$ & 6.6 & \\
\hline $43.409828 \pm 0.000094$ & $0.157 \pm 0.007$ & $0.9349 \pm 0.0279$ & 14.5 & 12 \\
\hline $44.356363 \pm 0.000223$ & $0.066 \pm 0.013$ & $0.9012 \pm 0.0557$ & 6.3 & \\
\hline $46.318147 \pm 0.000096$ & $0.153 \pm 0.007$ & $0.8000 \pm 0.0065$ & 14.2 & \\
\hline $46.456655 \pm 0.000235$ & $0.062 \pm 0.006$ & $0.6032 \pm 0.0175$ & 5.8 & \\
\hline $47.667615 \pm 0.000181$ & $0.081 \pm 0.006$ & $0.7426 \pm 0.0127$ & 7.6 & \\
\hline $48.238558 \pm 0.000322$ & $0.046 \pm 0.007$ & $0.4482 \pm 0.0228$ & 4.1 & \\
\hline $49.433689 \pm 0.000030$ & $0.491 \pm 0.008$ & $0.3511 \pm 0.0022$ & 29.4 & \\
\hline $50.002473 \pm 0.000149$ & $0.098 \pm 0.006$ & $0.8432 \pm 0.0115$ & 5.9 & 14 \\
\hline $50.999734 \pm 0.000177$ & $0.083 \pm 0.007$ & $0.7933 \pm 0.0123$ & 8.1 & \\
\hline $51.215952 \pm 0.000226$ & $0.065 \pm 0.006$ & $0.6727 \pm 0.0147$ & 6.6 & \\
\hline $52.672629 \pm 0.000043$ & $0.343 \pm 0.006$ & $0.1683 \pm 0.0032$ & 18.9 & \\
\hline $53.012605 \pm 0.000059$ & $0.248 \pm 0.006$ & $0.4302 \pm 0.0048$ & 14.0 & \\
\hline $53.173059 \pm 0.000051$ & $0.286 \pm 0.006$ & $0.0920 \pm 0.0038$ & 16.0 & \\
\hline $54.333293 \pm 0.000105$ & $0.140 \pm 0.007$ & $0.4882 \pm 0.0067$ & 13.7 & \\
\hline $55.076921 \pm 0.000137$ & $0.107 \pm 0.007$ & $0.7837 \pm 0.0089$ & 9.7 & \\
\hline $56.332132 \pm 0.000057$ & $0.258 \pm 0.006$ & $0.8862 \pm 0.0046$ & 12.2 & \\
\hline $56.337529 \pm 0.000080$ & $0.183 \pm 0.007$ & $0.6321 \pm 0.0054$ & 8.6 & \\
\hline $56.383938 \pm 0.000092$ & $0.160 \pm 0.007$ & $0.6144 \pm 0.0060$ & 7.6 & \\
\hline $56.700889 \pm 0.000030$ & $0.491 \pm 0.007$ & $0.9475 \pm 0.0021$ & 23.3 & 16 \\
\hline $59.709581 \pm 0.000095$ & $0.155 \pm 0.006$ & $0.4958 \pm 0.0066$ & 9.4 & \\
\hline $59.741600 \pm 0.000033$ & $0.442 \pm 0.012$ & $0.2427 \pm 0.0043$ & 26.7 & \\
\hline $59.746637 \pm 0.000155$ & $0.095 \pm 0.013$ & $0.4808 \pm 0.0097$ & 5.7 & \\
\hline $59.815711 \pm 0.000114$ & $0.129 \pm 0.007$ & $0.7050 \pm 0.0076$ & 7.8 & \\
\hline $63.180209 \pm 0.000074$ & $0.199 \pm 0.009$ & $0.5228 \pm 0.0058$ & 12.8 & 18 \\
\hline $63.290656 \pm 0.000035$ & $0.420 \pm 0.007$ & $0.7557 \pm 0.0025$ & 26.9 & \\
\hline $66.696167 \pm 0.000283$ & $0.052 \pm 0.007$ & $0.9038 \pm 0.0236$ & 6.5 & \\
\hline
\end{tabular}

and extracts additional data for a studied star from the public astronomical databases (TIC, SIMBAD, GALAH). The software creates images and a list of identified periodicities, and stores all the information in a final report.

The code PERIOD04 (version 1.2.9) developed by Lenz \& Breger (2005) has been specially designed to carry out a statistical analysis of large astronomical data sets with significant gaps and is a powerful tool for the identification and evaluation of periodic signals in long time series of stellar flux measurements. It has been recently modified by one of us (PL) to work in the batch mode such that it can be launched automatically from a script with a list of specified parameters. The significant peaks in the TESS light curve of HD 27463 have been extracted using the standard procedure of iterative pre-whitening and optimized using a multi-frequency least-squares fitting procedure (Lenz \& Breger 2005). This analysis yielded the detection of multiple frequencies with amplitudes having a significant signal-to-noise ratio. Significant peaks are defined as those with an amplitude larger than four times the level of noise remaining after pre-whitening the signal at each studied frequency.

Finally, the combined light curve was fitted taking into account all detected frequencies using the least-squares procedure and the obtained frequencies, amplitudes and phases are shown respectively in the first, second and third columns of Table 1 . The presented uncertainties are the largest ones obtained from the error matrix produced by the Levenberg-Marquardt non-linear least-squares fitting procedure and from a Monte Carlo simulation of a signal with the detected frequencies (Lenz \& Breger 2005). They do not take into account the estimation errors reported for the flux measurements. ${ }^{4}$

${ }^{4}$ Consideration of the observation errors during the Fourier analysis reveals no significant changes in the derived estimates of frequency or amplitude of the detected signals. Meanwhile it leads to changes of the best-fitting 
Table 2. Fundamental stellar parameters derived for HD 27463.

\begin{tabular}{lccc}
\hline Parameter & TIC, & \multicolumn{2}{c}{ This article } \\
& SIMBAD & Balmer lines & Pulsations \\
\hline$T_{\text {eff }}, \mathrm{K}$ & $8700 \pm 200^{a}$ & $8700 \pm 100$ & $8800 \pm 100$ \\
$\log g$ & $3.9 \pm 0.3^{a}$ & $3.9 \pm 0.2$ & $3.89 \pm 0.05$ \\
{$[\mathrm{M} / \mathrm{H}]$} & & $0.3 \pm 0.1$ & \\
$v \sin i, \mathrm{~km} \mathrm{~s}^{-1}$ & & $27 \pm 2$ & \\
$v_{\mathrm{r}}, \mathrm{km} \mathrm{s}^{-1}$ & $26.5 \pm 4.8^{b}$ & $22 \pm 2^{c}$ & \\
$L_{\star}, \mathrm{L}_{\odot}$ & $38.7 \pm 10.8^{a}$ & & $42.5 \pm 0.6$ \\
$R_{\star}, \mathrm{R}_{\odot}$ & $2.8 \pm 0.4^{a}$ & & \\
$M_{\star}, \mathrm{M}_{\odot}$ & $2.2 \pm 0.4^{a}$ & & $2.4 \pm 0.1$ \\
$v_{\text {eq }}, \mathrm{km} \mathrm{s}^{-1}$ & & $50 \pm 7$ & $47 \pm 5$ \\
$i, \mathrm{deg}$ & & $33 \pm 8$ & \\
age, $10^{8} \mathrm{yr}$ & & & $5.0 \pm 0.4$ \\
\hline
\end{tabular}

Notes. ${ }^{a}$ Stassun et al. $(2018,2019)$;

${ }^{b}$ Gontcharov (2006);

${ }^{c}$ in barycentric reference frame.

Using this approach, the uncorrelated uncertainties for the frequency and the phase have been derived (Montgomery \& O'Donoghue 1999). The fourth column of Table 1 contains the signal-to-noise ratio calculated for each mode at the derived frequency based on the simulation of white noise using the dispersion of residual data left after the pre-whitening procedure (Lenz \& Breger 2005). Meanwhile the fifth column of Table 1 specifies the radial order of $\ell=0$.

\section{STUDY OF FUNDAMENTAL STELLAR PARAMETERS}

Some fundamental stellar parameters of HD 27463 are taken from public astronomical databases (TIC, SIMBAD ${ }^{5}$ see Table 2). The effective temperatures reported in TIC has been derived using a new empirical relation between $T_{\mathrm{eff}}$ and Gaia $G_{\mathrm{BP}}-G_{\mathrm{RP}}$ colours, based on a set of 19962 stars (Stassun et al. 2019). The stellar radius was computed using the Gaia parallaxes employing the standard expression derived from the Stefan-Boltzmann law. Based on the obtained values of effective temperature and stellar radius one can calculate the respective bolometric luminosity, $L_{\mathrm{bol}}$. More details on the procedure of determination of the fundamental stellar parameters reported in TIC and its estimation errors can be found in Stassun et al. (2018, 2019).

Our estimates of the effective temperature, surface gravity, metallicity, radial velocity and $v \sin i$ have been derived by fitting the non-normalized profiles of Balmer lines (see Fig. 4). The derived value of $v \sin i=27 \pm 2 \mathrm{~km} \mathrm{~s}^{-1}$ has been confirmed by analysis of several Fe II line profiles visible in the two FEROS spectra of this star.

We assume here that the high-amplitude periodic variability in the light curve with the lowest frequency and the associated first harmonic (see Table 1) correspond to the stellar rotation. Therefore, we have used the estimate of the stellar radius from the TIC and the spectroscopic value of $v \sin i$ to derive the equatorial velocity, $v_{\mathrm{eq}}$, and inclination angle, $i$, of the rotational axis with respect to the line of sight (see Table 2).

phase derived for the weak signals and increases by an order of magnitude the errors obtained from the Monte Carlo simulation for the estimates of frequency, amplitude and phase.

${ }^{5}$ simbad.u-strasbg.fr/simbad/sim-id?Ident $=$ HIP19917

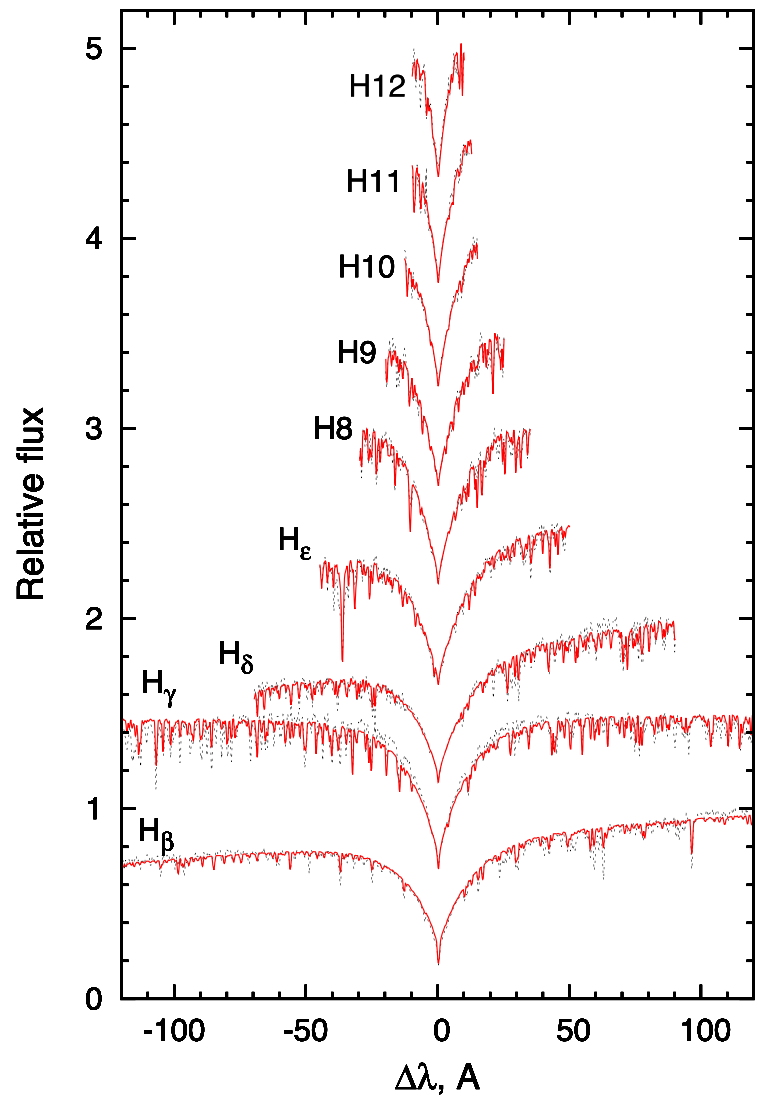

Figure 4. An example of fitting the observed Balmer line profiles (thick red line) of HD 27463 with a synthetic spectrum (thin dotted line) that corresponds to $T_{\text {eff }}=8700 \pm 100 \mathrm{~K}, \log g=3.9 \pm 0.1,[\mathrm{M} / \mathrm{H}]=0.3 \pm 0.1$ $\left(\chi^{2}=1.081\right)$. The best fit is obtained for the radial velocity $v_{\mathrm{r}}=22 \pm 2 \mathrm{~km}$ $\mathrm{s}^{-1}$ and $v \sin i=27 \pm 2 \mathrm{~km} \mathrm{~s}^{-1}$ (see Table 2). The observed Balmer line profiles are shifted by 0.5 for visibility.

\subsection{Analysis of Balmer line profiles}

The Balmer line profiles of HD 27463 have been fitted employing the FITSB2 code (Napiwotzki et al. 2004) to derive best-fitting parameters ( $T_{\text {eff }}, \log g$ and metallicity) of the stellar atmosphere. This code uses grids of synthetic fluxes simulated with the code PHOENIX (Hauschildt et al. 1997) for different values of $T_{\text {eff }}, \log g$ and metallicity. During this procedure the code takes into account the level of nearby continuum and the intensity of some strong metallic lines that are present in the Balmer wings. The FITSB 2 code does not perform an abundance analysis for each chemical element, but it rather results in an estimate of metallicity that serves as an average measure of the abundance of metals. For this reason, the spectral lines of most metals are not well fitted in Fig. 4.

Specifically, in this study we use the grids of synthetic fluxes ${ }^{6}$ simulated by Husser et al. (2013) for models with different metallicities and abundances of the $\alpha$-elements $(\mathrm{O}, \mathrm{Ne}, \mathrm{Mg}, \mathrm{Si}, \mathrm{S}, \mathrm{Ar}, \mathrm{Ca}$, and Ti) employing version $16^{7}$ of the PHOENIX code (Hauschildt \& Baron 1999). These grids were computed with a spectral resolution

\footnotetext{
${ }^{6}$ The grids of synthetic spectra are available at http://phoenix.astro.physik uni-goettingen.de/.

${ }^{7}$ Compared to the previous version, version 16 of the PHOENIX code employs an updated list of atomic and molecular lines and uses a new equation of state to simulate a model of the stellar atmosphere and the synthetic emerging flux (Husser et al. 2013) of the PHOENIX code.
} 
of $R=500000$, which we reduced to $R=50000$ during their transformation to the format read by the FITSB 2 code (Khalack et al. 2017). This latter resolution is close to the spectral resolution of FEROS (see Section 2).

Two available FEROS spectra of HD 27463 were analysed individually and resulted in similar values of effective temperature, surface gravity and metallicity. The best-fitting results obtained for one spectrum of HD 27463 that corresponds to the lowest $\chi^{2}$ are shown in Table 2 and Fig. 4. This figure shows that the obtained best fit approximates the analysed Balmer line profiles relatively well even taking into account the contamination of Balmer wings by some metal lines. The fitting procedure has been carried out for different values of rotational velocity by including rotational broadening to the theoretical line profiles before the fitting of Balmer line profiles. In this case, the best-fitting value of $v \sin i$ is partially constrained by the metal lines present in the Balmer line wings. This approach allowed us to find the value that minimizes the discrepancy between the observed and simulated Balmer line profiles for $v \sin i$ $=27 \pm 2 \mathrm{~km} \mathrm{~s}^{-1}$ (see Table 2). The derived value of $v \sin i$ has been confirmed by analysis of several Fe II line profiles visible in the two FEROS spectra of this star.

\subsection{Analysis of stellar pulsations}

We have calculated a grid of stellar structure and evolution models using MESA version 11554 (Paxton et al. 2011, 2013, 2015, 2018, 2019), including the effects of convective overshoot. Model masses ranged from 2.0 to $2.6 \mathrm{M}_{\odot}$ in $0.1 \mathrm{M}_{\odot}$ increments, with rotation rates on the ZAMS (Zero Age Main Sequence) between 35 and $100 \mathrm{~km} \mathrm{~s}^{-1}$ taking into account the derived value of the equatorial velocity (see Table 2 ). We used a scaled solar metallicity of $Z$ $=0.0244$ for all models to account for the inferred spectroscopic metallicity of the star. Inside the convective core, convective mixing is calculated using mixing-length theory (Böhm-Vitense 1958) with a mixing length $\alpha=1.73$ (see e.g. Montalbán et al. 2004). Convective overshoot was included above the core using the diffusive exponential overshoot model described by Herwig (2000):

$D_{\mathrm{ov}}=D_{0} \exp \left(\frac{-2 r}{f_{\mathrm{ov}} H_{\mathrm{P}}}\right)$,

where $D_{0}$ is the diffusion coefficient at the convective boundary, $r$ is the radial distance from the core boundary, and $H_{\mathrm{P}}$ is the pressure scale height at the core boundary. The amount of overshoot is defined in terms of a fraction of the pressure scale height, $H_{\mathrm{P}}$. In our models, the amount of overshoot ranged from $f_{\text {ov }}=0$ to 0.04 in increments of 0.01 . As many of our best-fitting models were found to have overshoot between 0.01 and 0.03 , this region of the grid was subsequently refined with steps of 0.002 . The models were evolved from the pre-main sequence through the end of the main sequence. Detailed models were saved at least every 20 time steps, and more frequently during certain evolutionary phases. The time steps were variable, and determined by the requirement that the relative change in the stellar structure be less than $10^{-4}$ from one time step to the next. The evolution tracks for the zero-overshoot models are shown in Fig. 5.

We used GYRE (Townsend \& Teitler 2013) to calculate linear adiabatic pulsation frequencies for each main-sequence model with $3.92<\log T_{\text {eff }}<3.95$, consistent with both the temperature from the TIC and that derived from the Balmer lines in this work. We calculated model frequencies between 40 and $70 \mathrm{~d}^{-1}$ for $\ell=0,1$, 2 , and 3. Initially, we calculated frequencies for $m=0$ modes only.

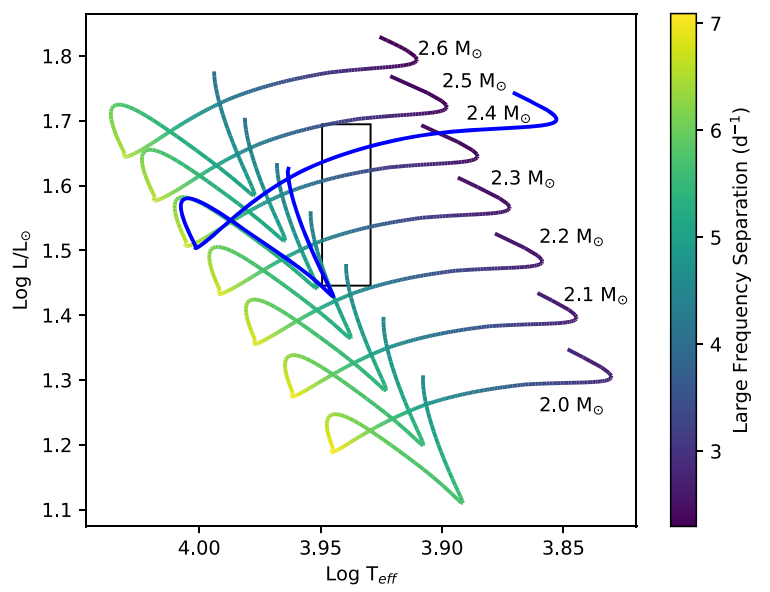

Figure 5. The main-sequence evolution tracks for MESA models from 2.0 to $2.6 \mathrm{M}_{\odot}$, all with zero convective overshoot and a metallicity derived from spectroscopy of HD 27463, are shown in colours that correspond to expected values of the large frequency separation. The evolution track for the best-fitting model with $M_{\star}=2.4 \mathrm{M}_{\odot}, f_{\text {ov }}=0.014$ is shown in blue. The box indicates the $1 \sigma$ spectroscopic uncertainty box in the observed location of HD 27463.

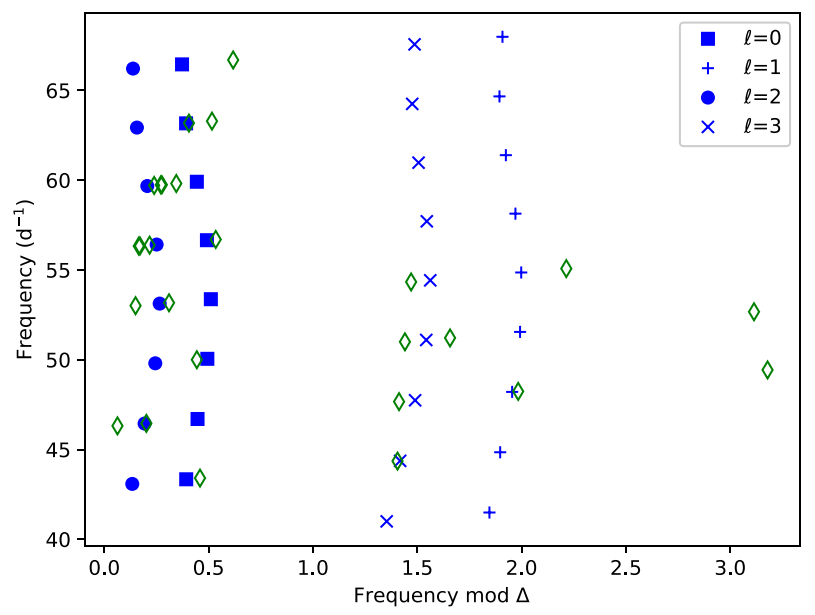

Figure 6. The echelle diagram for the best-fitting model and the observed frequencies, plotted using the inferred large frequency separation of $\Delta v=$ $3.304 \mathrm{~d}^{-1}$. The blue points show the model frequencies for $\ell=0$ (squares), $\ell=1$ (pluses), $\ell=2$ (circles), and $\ell=3$ (crosses), while the green open diamonds specify the data derived from observations. The model frequencies plotted here correspond to radial orders of $n=11-19$.

Once we had determined a small set of models that were good fits to the observations, we calculated the $m \neq 0$ modes for comparison to the observed rotational splitting.

We used the 26 frequencies from Table 1 , all of which are greater than $43 \mathrm{~d}^{-1}$ and appear to correspond to stellar pulsation modes to determine the large frequency separation

$\Delta v_{0}=<v_{n+1, \ell}-v_{n, \ell}>$.

Given the spectroscopically determined $T_{\text {eff }}$ value used as an input constraint to the modelling, the best-fitting large frequency separation is approximately $3.3 \mathrm{~d}^{-1}$, which produces two clear ridges in the echelle diagram, as shown in Fig. 6. We then compared this derived large frequency separation to the values calculated for the models in our grid. The absolute value of the difference between the model and the observed large frequency separation 


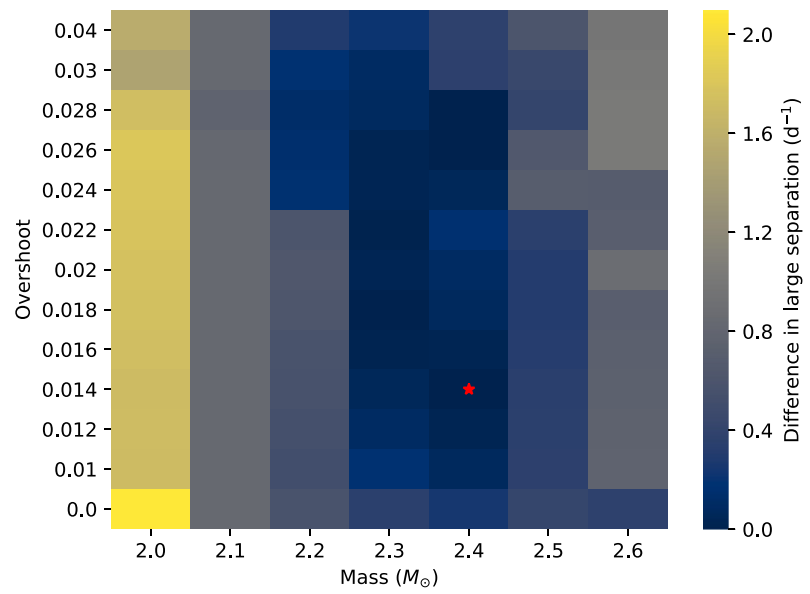

Figure 7. The absolute value of the difference in the large frequency separation for the models in our grid compared to the observed value, shown as a function of mass and overshoot. Based on the difference in the large frequency separation and comparison to echelle diagrams, the best-fitting model (red star) was determined to have $M_{\star}=2.4 \mathrm{M}_{\odot}$ and $f_{\text {ov }}=0.014$.

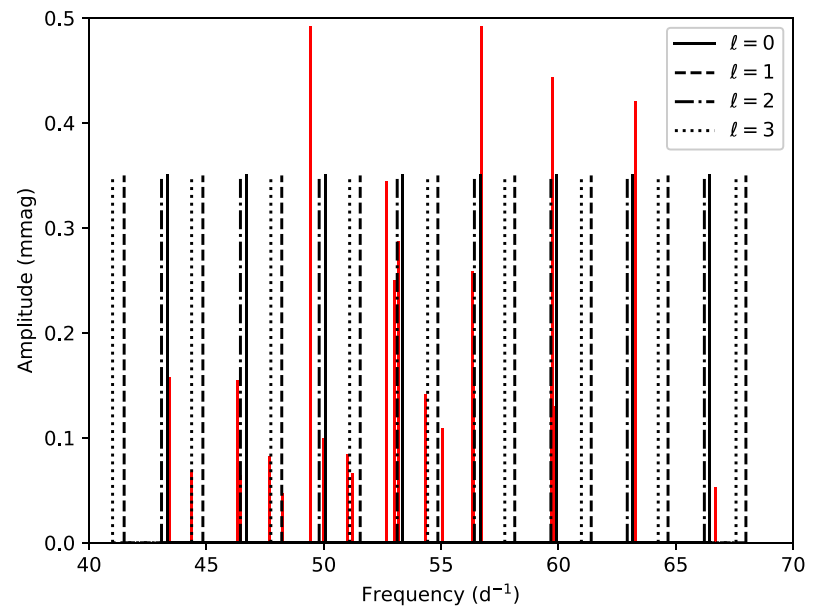

Figure 8. Individual frequency matches for the best-fitting model. The red lines are the observed frequencies plotted versus amplitude, while the black lines are the $\ell=0$ (solid), $\ell=1$ (dashed), $\ell=2$ (dot-dashed), and $\ell=3$ (dotted) model frequencies.

is shown in Fig. 7. A number of models with masses between 2.2 and $2.5 \mathrm{M}_{\odot}$ have reasonably good fits to the large frequency separation. For models with the smallest difference in the large frequency separation, the echelle diagrams were plotted. Not all models with a large frequency separation of $3.3 \mathrm{~d}^{-1}$ were good fits to the observed echelle diagram, so we also compared individual frequency fits, as shown in Fig. 8.

HD 27463 has been analysed recently by Bedding et al. (2019) who have performed mode identification using the echelle diagram and obtained a large frequency separation of $6.91 \mathrm{~d}^{-1}$, more than twice our result. Our models show that HD 27463 is an evolved $\delta$ Scuti star with an age of $5 \times 10^{8} \mathrm{yr}$ and an effective temperature that is similar to the one found from the analysis of Balmer line profiles (see Table 2). In Fig. 5, the $1 \sigma$ spectroscopic uncertainty box that specifies the observed location of HD 27463 on the Hertzsprung-Russell diagram is constructed taking into account uncertainties provided by the TIC for the estimates of stellar luminosity and effective temperature (Stassun et al. 2018, 2019).

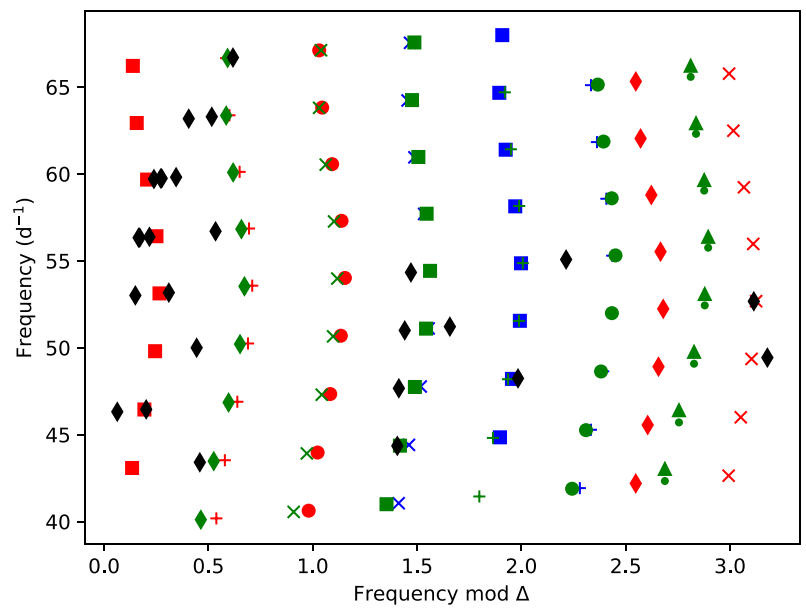

Figure 9. The mode splitting in the $\ell=1$ (blue), 2 (red), 3 (green) modes for the best-fitting model. The $m=-3,-2,-1,0,1,2$ and 3 frequencies are denoted by triangles, diamonds, crosses, squares, pluses, circles, and dots respectively. The black diamonds indicate the observed frequencies. The range of radial orders shown is the same as in Fig. 6.

Fig. 5 clearly demonstrates that the large frequency separation around $\Delta v_{0}=6.9 \mathrm{~d}^{-1}$ is a characteristic of very young stars (see also Bedding et al. 2019).

We have repeated our fitting procedure assuming a large frequency separation of $6.91 \mathrm{~d}^{-1}$ derived by Bedding et al. (2019). In this case, we found that the best-fitting model has a large frequency separation of $6.707 \mathrm{~d}^{-1}$ and is a $2.0 \mathrm{M}_{\odot}$ model with a core overshoot of 0.03 and a ZAMS equatorial rotation velocity of $40 \mathrm{~km} \mathrm{~s}^{-1}$. The echelle diagram and frequency fit for this model are shown in Figs A1 and A2 respectively. The model corresponds to a young star that is effectively on the ZAMS. The derived $\chi^{2}$ for the best frequency fit is an order of magnitude larger than our best fit with $\Delta v_{0}=3.3 \mathrm{~d}^{-1}$, which takes into account the spectroscopic constraints on temperature and metallicity (see Figs 5, 6, 7 and 8). This finding clearly indicates that the large frequency separation is not a unique factor on which we should rely to determine the fundamental stellar parameters. The spectroscopy and specifically spectroscopic estimates of $T_{\text {eff }}$ and metallicity are needed to break degeneracies amongst fundamental parameters when performing forward seismic modelling (see Aerts et al. 2018; Buysschaert et al. 2018).

Based on our simulations, the best-fitting model was determined to have a large frequency separation of $3.304 \mathrm{~d}^{-1}$, and is compared to the observations in Fig. 6 . This model has a mass of $2.4 \mathrm{M}_{\odot}$, a ZAMS rotation velocity of $47 \pm 5 \mathrm{~km} \mathrm{~s}^{-1}$, and an overshoot of $f_{\text {ov }}=0.014$. The derived values of effective temperature, $T_{\text {eff }}=$ $8800 \pm 100 \mathrm{~K}$, and surface gravity, $\log g=3.89 \pm 0.05$, are in good agreement with the spectroscopic measurements (see Table 2). The stellar luminosity of $42.5 \mathrm{~L}_{\odot}$ derived from the best-fitting model is comparable to the value reported in the TIC.

During the simulation of stellar structure, the core hydrogen fraction evolves with the star and is one of the output parameters provided by the code MESA (Paxton et al. 2011, 2013, 2015, 2018, 2019). Assuming that the initial core hydrogen fraction is $X_{\mathrm{c}}=0.7$, our best-fitting model was determined to have an age of $5 \times 10^{8}$ $\mathrm{yr}$, which corresponds to $X_{\mathrm{c}}=0.33$. These parameters are derived from the best-fitting model to the echelle diagram (see Fig. 9).

The amount of overshooting that we found in this model is similar to that found for KIC 10526294 (Moravveji et al. 2015), 
and slightly lower than that found for KIC 7760680 (Moravveji et al. 2016). However, both of these stars are late-type B stars, and show g-mode pulsations typical of slowly pulsating B (SPB) stars. Similar studies of a magnetic B-type star (HD 43317) found a much lower convective overshoot $\left(f_{\text {ov }}=0.004\right)$ and the authors conclude that convective mixing is suppressed by the magnetic field (Buysschaert et al. 2018). More recently, Mombarg et al. (2019) performed ensemble asteroseismic modelling of gravity modes in $37 \gamma$ Doradus stars observed by the Kepler space mission. Mombarg et al. (2019) found best-fitting diffusive exponential overshooting values that range between 0 and 0.03 for these main-sequence intermediate-mass stars.

Once a best-fitting model was established, we calculated rotational splitting in the $\ell=1,2$ and 3 modes to see if this provided matches for some of the unmatched frequencies in the echelle diagram, since rotation also has a significant impact on pulsation mode frequencies (Aerts et al. 2010). As shown in Fig. 9, the observed mode splitting agrees well with our model calculations. For a model that is initially rotating at $100 \mathrm{~km} \mathrm{~s}^{-1}$, the present-day rotation rate has dropped to $47 \mathrm{~km} \mathrm{~s}^{-1}\left(\Omega / \Omega_{\text {crit }}=0.12\right)$ at $X_{\mathrm{c}}$ $=0.33$.

\section{DISCUSSION}

The detected photometric variability of HD 27463 with a period of $P=2.834274 \pm 0.000008 \mathrm{~d}$ (see Table 1 and Fig. 3) can be explained in terms of stellar rotation with co-rotating surface abundance/brightness patches, likely due to the presence of a surface magnetic field. In a hydrodynamically stable stellar atmosphere even a relatively weak magnetic field can amplify the atomic diffusion and lead to horizontal and vertical stratification of chemical abundances (Alecian \& Stift 2010; Stift \& Alecian 2012, 2016). Usually the magnetic CP stars host magnetic fields that are dominated by a dipolar component with strengths of the order of several $\mathrm{kG}$ or less, which remains stable over long time-scales (Silvester et al. 2014; Kochukhov et al. 2019). The magnetically sensitive line profiles Fe II $6147 \AA$, $6149 \AA$ visible in the FEROS spectra of HD 27463 are not visually split due to the Zeeman effect. Taking into account the derived value of $v \sin i=27 \pm 2 \mathrm{~km} \mathrm{~s}^{-1}$, it seems that the magnetic field modulus might be of the order of a few $\mathrm{kG}$ in the stellar atmosphere of this star. It is significant enough to cause the formation of abundance patches in the stellar atmosphere of HD 27463 taking into account its spectral classification as an Ap $\mathrm{EuCr}(\mathrm{Sr})$ star (Houk \& Cowley 1975). A preliminary analysis of the two FEROS spectra shows that the profiles of $\mathrm{Si}$ and $\mathrm{Ca}$ lines are strongly variable, which confirms the Ap nature of this star. The patches of enhanced abundance of different metals usually are located close to the magnetic poles or along the magnetic equator (Lüftinger et al. 2010; Kochukhov et al. 2019; Mathys et al. 2019) and contribute to the variability of spectral line profiles and the photometric flux in phase with the stellar rotation. Assuming the oblique magnetic rotator model (Stibbs 1950) in the case of HD 27463 the overabundance patches will generate a signal at the first harmonics of the rotational frequency. The derived pattern of stellar variability in the low-frequency domain is in good accordance with the predictions of the oblique magnetic rotator model (see Fig. 3).

The lower panel of Fig. 3 indicates that HD 27463 shows highfrequency pulsations $\left(40<v<70 \mathrm{~d}^{-1}\right)$ with amplitudes less than $0.5 \mathrm{mmag}$. Such high-frequency pulsation modes are typically found in hotter $\delta$ Scuti stars (Smalley et al. 2017; Bowman \& Kurtz 2018). This is because the heat-engine driving mechanism operating in the He II ionization zone is closer to the surface and is more
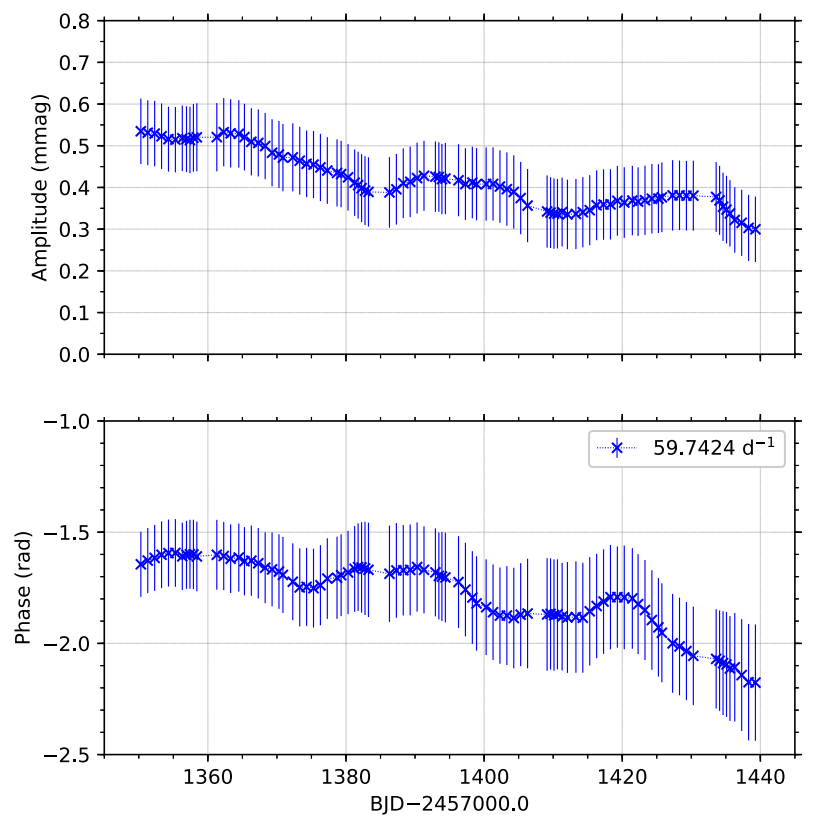

Figure 10. Variability of the amplitude and phase with time for stellar pulsation detected at the frequency $v=59.7424 \mathrm{~d}^{-1} .1 \sigma$ uncertainties are determined from the least-squares fit.

efficient at exciting higher radial orders, hence higher frequencies, in hotter $\delta$ Scuti stars (Pamyatnykh 2000; Dupret et al. 2019). Cunha et al. (2019) and Sikora et al. (2019) have also classified this star as a suspected $\delta$ Scuti variable. Therefore, our spectroscopic parameters and subsequent modelling results are in agreement with this theoretically predicted and observed relationship.

Many $\delta$ Scuti stars are known to show a long-term variability of their pulsation amplitudes and frequencies (Breger 2000; Bowman 2017). To study possible amplitude variability in HD 27463, we employed the methodology of Bowman et al. (2016), such that the amplitude and phase of a pulsation mode was tracked at fixed frequency using linear least-squares over the duration of the TESS light curve. To achieve this, the light curve of HD 27463 was divided into bins of $50 \mathrm{~d}$ with a step of $1 \mathrm{~d}$, and amplitude and phase of a pulsation mode were optimized using linear least-squares in each bin for the frequency determined using the entire light curve. In our analysis, the zero-point of the time used in the calculation of phases was chosen as the approximate midpoint of the entire TESS light curve (i.e. BJD 2457 1340.0). The $1 \sigma$ uncertainties for the amplitude and phase values were obtained from the least-squares fit.

Employing this technique we were able to detect marginal amplitude and phase modulation given the relatively large uncertainties for the pulsation modes in HD 27463. An example of the amplitude and phase modulation for the pulsation mode frequency at $59.2474 \mathrm{~d}^{-1}$ is shown in Fig. 10. Other pulsation modes with a relatively high amplitude (see Table 1) show similar behaviour. Our detection of amplitude and phase modulation for pulsation modes with relatively high amplitudes is in agreement with the findings of Bowman et al. (2016) that amplitude modulation is common in $\delta$ Scuti stars with long-term amplitude modulation occurring on time-scales much longer than the rotation period. Some pulsation modes in HD 27463 have very small amplitudes. In this case, it is difficult to search for an amplitude modulation taking into account 
that the error-bars derived from a non-linear least-squares fit are of the order of $0.01 \mathrm{mmag}$.

We have also analysed the 26 frequencies of detected stellar pulsations located in the frequency range between 40 and $70 \mathrm{~d}^{-1}$ (see Table 1) that result in the best-fitting large frequency separation around $3.3 \mathrm{~d}^{-1}$, which is used as a constraint to model pulsation modes (see Fig. 6). The grid of stellar evolution models has been calculated with MESA version 11554 (Paxton et al. 2011, 2013, 2015, 2018, 2019) including the effects of convective overshoot and metallicity, while the simulation of linear adiabatic pulsation frequencies for each main-sequence model has been carried out using the code GYRE (Townsend \& Teitler 2013). Our best-fitting model corresponds to an overshoot parameter of $f_{\mathrm{ov}}=0.014$ and results in values of global stellar parameters that are very close to those reported in the TIC and to those derived in this paper from the simulation of Balmer line profiles (see Table 2). This model was determined to have an age of $5.0 \pm 0.4 \times 10^{8} \mathrm{yr}$, which corresponds to a core hydrogen fraction of 0.33 .

We have found that the large frequency separation is not a sensitive function of the convective core overshoot, as shown in Fig. 7. The spectroscopic constraints on fundamental parameters, especially $T_{\text {eff }}$ and metallicity, in addition to the observed large frequency separation serve to narrow down the number of models, which must then be compared to the observed echelle ridges (see Figs 6,9) and the individual frequencies (see Fig. 8) to determine the best-fitting parameters.

The radial orders inferred from this work are high for $\delta$ Scuti stars, which are typically observed to have low $(1 \leq n \leq 7)$ radial orders. It has been shown by Antoci et al. (2014) that moderate (7 $\leq n \leq 15$ ) radial orders can be excited in $\delta$ Scuti stars by means of turbulent pressure, although this pulsation excitation mechanism is most efficient in the centre of the classical instability strip. HD 27463 has been shown in this work to be closer to the predicted blue edge of the classical instability strip instead of the centre, with an effective temperature of approximately $8700 \mathrm{~K}$. Nevertheless, we conclude that turbulent pressure must play a role, at least in part, in the excitation of such high-frequency pulsation modes in HD 27463.

Taking into account that HD 27463 is a long period ( $~ 370 \mathrm{yr})$ visual binary with a separation of $\sim 0.3 \operatorname{arcsec}$ (Baize \& Petit 1989; Hartkopf et al. 2012) we cannot exclude that the detected pulsations are produced by the weaker secondary companion. Having its visual magnitude only 0.43 less than the one of the Ap primary, this companion can also fall in the $\delta$ Scuti instability region and contribute to the observed by TESS light curve of HD 27463. Comparison of the two FEROS spectra does not show clear evidence of contribution from the secondary companion. Nevertheless, to estimate the parameters of this binary and to clarify the nature of the secondary companion we require acquisition of additional highresolution and high signal-to-noise spectra of HD 27463.

We have confirmed here that HD 27463 is an Ap star. Excitation of high-radial-order magneto-acoustic modes is expected and is observed in some Ap stars (the roAp stars), which possess a global magnetic field usually of dipolar structure (Kurtz 1982; Kurtz et al. 2006; Bigot \& Kurtz 2011). Therefore, higher-resolution spectroscopic and/or spectropolarimetric observations are also required to detect the presence of a strong large-scale magnetic field in HD 27463 to say conclusively whether the star is just a chemically peculiar $\delta$ Scuti star, or whether it can be called a roAp star. Our estimation of the upper limit on the magnetic field modulus is not in contradiction with the results of Saio (2005), whose models require polar magnetic field strengths of $\sim 0.8 \mathrm{kG}$ or more to suppress $\delta$ Scuti pulsations. However, a precise measurement of the magnetic field in HD 27463 is required to determine the exact driving mechanism in this star and the influence of the magnetic field on the pulsation modes.

The distinction between roAp stars and chemically peculiar $\delta$ Scuti stars based on photometric data alone is becoming less clear (e.g. Balona et al. 2019). Typically, an Ap star showing rapid pulsations was historically sufficient to classify a roAp star. However, the roAp stars can show rotationally split sidelobes in the amplitude spectra of their light curves, which are a result of oblique pulsation (e.g. Kurtz 1982; Bigot \& Kurtz 2011). These sidelobes arise as the pulsation axis in the roAp stars is closely aligned with the magnetic axis, which is inclined to the rotational one. To detect the rotational sidelobes, the line of sight to the pulsation needs to be favourable, and the rotation period shorter than the observation period. There is no indication of rotationally split sidelobes in HD 27463 , which could mean that we are unfortunate in the geometry or that the pulsations are not oblique, i.e. the pulsation and rotation axes are aligned as in chemically normal $\delta$ Scuti stars.

To conclusively say whether HD 27463 is a chemically peculiar $\delta$ Scuti star or a roAp star and to clarify the nature of the secondary companion, extensive high-resolution spectroscopic and/or spectropolarimetric observations are required. These data, along with further detailed modelling of the star that takes into account the magnetic field, are needed to distinguish between these two classes of pulsator, and to investigate to what extent the magnetic field plays a role in this star and others with similar characteristics. Nonetheless, the rich frequency spectrum of HD 27463 including radial and non-radial modes spanning several radial orders demonstrates the exciting prospects for asteroseismic studies of intermediate-mass stars with TESS.

\section{ACKNOWLEDGEMENTS}

The authors are grateful to Profs. Don Kurtz, Timothy Bedding, Daniel Huber and the anonymous referee for useful suggestions and comments that helped to improve this work significantly. VK, CL, ADU and GAW acknowledge support from the Natural Sciences and Engineering Research Council of Canada (NSERC). O. Kobzar and VK are grateful to the Faculté des Études Supérieures et de la Recherche and to the Faculté des Sciences de l'Université de Moncton for the financial support for this research. OK acknowledges support from the Swedish Research Council (project 621-20145720) and the Swedish National Space Board (projects 185/14, 137/17). DLH acknowledges financial support from the Science and Technology Facilities Council (STFC) via grant ST/M000877/1. This paper includes data collected by the TESS mission. Funding for the TESS mission is provided by the NASA Explorer Program. Funding for the TESS Asteroseismic Science Operations Centre is provided by the Danish National Research Foundation (grant agreement no. DNRF106), ESA PRODEX (PEA 4000119301) and the Stellar Astrophysics Centre (SAC) at Aarhus University. We thank the TESS and TASC/TASOC teams for their support of the present work. This research has made use of the SIMBAD database, operated at CDS, Strasbourg, France. Some of the data presented in this paper were obtained from the Mikulski Archive for Space Telescopes (MAST). STScI is operated by the Association of Universities for Research in Astronomy, Inc., under NASA contract NAS5-2655. The research leading to these results has received funding from the European Research Council (ERC) under the European Union's Horizon 2020 research and innovation programme (grant agreement no. 670519: MAMSIE). This study is 
also partially based on data obtained from the ESO Science Archive Facility under request number Khalack399537.

\section{REFERENCES}

Aerts C., Christensen-Dalsgaard J., Kurtz D. W., 2010, Asteroseismology. Springer, Berlin

Aerts C. et al., 2018, ApJS, 237, 15

Alecian G., Stift M. J., 2010, A\&A, 516, 53

Antoci V. et al., 2014, ApJ, 796, 118

Babcock H. W., 1958, ApJ, 128, 228

Baize P., Petit M., 1989, A\&AS, 77, 497

Balona L. A., Holdsworth D. L., Cunha M. S., 2019, MNRAS, 487, 2117

Bedding T. R. et al., 2019, submitted

Bigot L., Kurtz D. W., 2011, A\&A, 536, 73

Böhm-Vitense E., 1958, Z. Astrophys., 46, 108

Bowman D. M., 2017, Amplitude Modulation of Pulsation Modes in Delta Scuti Stars, Springer Theses series. Springer, Berlin

Bowman D. M., Kurtz D. W., 2018, MNRAS, 476, 3169

Bowman D. M., Kurtz D. W., Breger M., Murphy S. J., Holdsworth D. L., 2016, MNRAS, 460, 1970

Bowman D. M., Buysschaert B., Neiner C., Pápics P. I., Oksala M. E., Aerts C., 2018, A\&A, 616, A77

Breger M., 2000, MNRAS, 313, 129

Buysschaert B., Aerts C., Bowman D. M., Johnston C., Van Reeth T., Pedersen M. G., Mathis S., Neiner C., 2018, A\&A, 616, A148

Campante T. L. et al., 2016, ApJ, 830, 138

Cleveland W. S., 1979, J. American Statistical Association , 74, 829

Cunha M. S. et al., 2019, MNRAS, 487, 3523

David-Uraz A. et al., 2019, MNRAS, 487, 304

Dupret M. A., Grigahcène A., Garrido R., Gabriel M., Scuflaire R., 2004, A\&A, 414, L17

ESA, 1997, The Hipparcos and Tycho Catalogues (ESA, SP Series 1200). ESA, Noordwijk

Gontcharov G. A., 2006, Pis'ma Astron. Zh., 32, 844

Hartkopf W. I., Tokovinin A., Mason B. D., 2012, AJ, 143, 42

Hauschildt P. H., Baron E., 1999, J. Comput. Applied Math., 109, 41

Hauschildt P. H., Baron E., Allard F., 1997, ApJ, 483, 390

Herwig F., 2000, A\&A, 360, 952

Holdsworth D. L. et al., 2014, MNRAS, 439, 2078

Holdsworth D. L., Kurtz D. W., Smalley B., Saio H., Handler G., Murphy S. J., Lehmann H., 2016, MNRAS, 462, 876

Holdsworth D. L., Cunha M. S., Shibahashi H., Kurtz D. W., Bowman D. M., 2018, MNRAS, 480, 2976

Houk N., Cowley A. P., 1975, Michigan Spectral Survey, 1, 19

Husser T.-O. et al., 2013, A\&A, 553, 6

Jenkins J. M. et al. 2016, in Chiozzi G., Guzman J. C., eds, Proc. SPIE 9913, Software and Cyberinfrastructure for Astronomy IV, Vol. 9913. SPIE, id. 99133E

Joshi S., et al., 2016, A\&A, 590, A116

Kaufer A., Stahl O., Tubbesing S., Nørregaard P., Avila G., Francois P., Pasquini L., Pizzella A., 1999, Messenger, 95, 8

Khalack V., LeBlanc F., 2015a, AJ, 150, 2

Khalack V., LeBlanc F., 2015b, Advances Astron. Space Phys., 5, 3

Khalack V., Gallant G., Thibeault C., 2017, MNRAS, 471, 926
Kochukhov O., Shultz M., Neiner C., 2019, A\&A, 621, 47K

Kurtz D. W., 1978, Inf. Bull. Var. Stars, 1436, 1

Kurtz D. W., 1982, MNRAS, 200, 807

Kurtz D. W., Elkin V. G., Cunha M. S., Mathys G., Hubrig S., Wolff B., Savanov I., 2006, MNRAS, 372, 286

Lenz P., Breger M., 2005, Commun. Asteroseismology, 146, 53

Lüftinger T. et al., 2010, A\&A, 509, 43L

Martinez P., Kurtz D. W., 1994, MNRAS, 271, 129

Mathys G., Khalack V., Landstreet J., 2019, A\&A, submitted

Maury A. C., Pickering E. C., 1897, Ann. Harvard College Obser., 28, 1

Mombarg J. S. G., Van Reeth T., Pedersen M. G., Molenberghs G., Bowman D. M., Johnston C., Tkachenko A., Aerts C., 2019, MNRAS, 485, 3248

Montalbán J., D’Antona F., Kupka F., Heiter U., 2004, A\&A, 416, 1081

Montgomery M. H., O’Donoghue D., 1999, Delta Scuti Star Newsletter, 13, 28

Moravveji E., Aerts C., Pápics P. I., Triana S. A., Vandoren B., 2015, A\&A, 580, A27

Moravveji E., Townsend R. H. D., Aerts C., Mathis S., 2016, ApJ, 823, 130

Napiwotzki R. et al., 2004, in Hilditch R. W., Hensberge H., Pavlovski K., eds, ASP Conf. Ser. Vol. 318, Spectroscopically and Spatially Resolving the Components of the Close Binary Stars. Astron. Soc. Pac., San Francisco, p. 402

Pamyatnykh A. A., 2000, in Breger M., Montgomery M., eds, ASP Conf. Ser. Vol. 210, Delta Scuti and Related Stars. Astron. Soc. Pac., San Francisco. p. 215

Paxton B., Bildsten L., Dotter A., Herwig F., Lesaffre P., Timmes F., 2011, ApJS, 192, 3

Paxton B. et al., 2013, ApJS, 208, 4

Paxton B. et al., 2015, ApJS, 220, 15

Paxton B. et al., 2018, ApJS, 234, 34

Paxton B. et al., 2019, ApJS, 243, 10

Renson P., Catalano F. A., 2001, A\&A, 378, 113

Ricker G., Vanderspek R., 2018, Data Product from TESS Data Alert

Ricker G. R. et al., 2015, J. Astron. Telesc. Instrum. Syst., 1, 014003

Saio H., 2005, MNRAS, 360, 1022

Seabold S., Perktold J., 2010, in van der Walt S., Millman J., eds, Proc. 9th Python in Science Conf., SciPy 2010, Austin, Texas, p. 62

Sikora J. et al., 2019, MNRAS, 487, 4695

Silvester J., Kochukhov O., Wade G. A., 2014, MNRAS, 440, 182

Smalley B. et al., 2017, MNRAS, 465, 2662

Stassun K. G. et al., 2018, AJ, 156, 102

Stassun K. G. et al., 2019, AJ, 158, 138

Stibbs D. W. N., 1950, MNRAS, 110, 395

Stift M. J., Alecian G., 2012, MNRAS, 425, 2715

Stift M. J., Alecian G., 2016, MNRAS, 457, 74

Townsend R. H. D., Teitler S. A., 2013, MNRAS, 435, 3406

\section{APPENDIX A: DEGENERACY OF THE LARGE FREQUENCY SEPARATION}

The echelle diagram and frequency fit for the best-fitting model that has a large frequency separation of $6.707 \mathrm{~d}^{-1}$ and a stellar mass of $2.0 \mathrm{M}_{\odot}$ with a core overshoot of 0.03 and a ZAMS equatorial rotation velocity of $40 \mathrm{~km} \mathrm{~s}^{-1}$. 


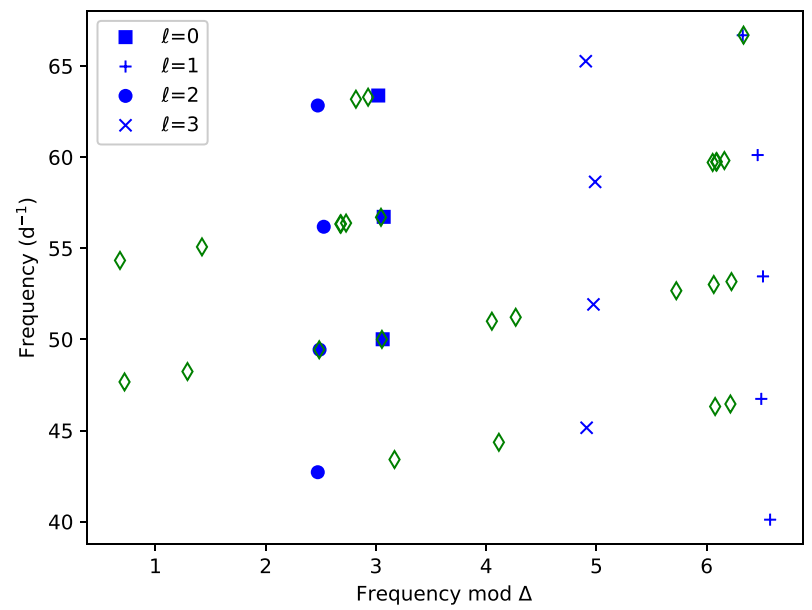

Figure A1. The echelle diagram for the best-fitting model and the observed frequencies, both plotted with a large frequency separation of $\Delta v_{0}=6.707 \mathrm{~d}^{-1}$. The blue points show the model frequencies for $\ell=$ 0 (squares), $\ell=1$ (pluses), $\ell=2$ (circles), and $\ell=3$ (crosses), while the green open diamonds specify the data derived from observations.

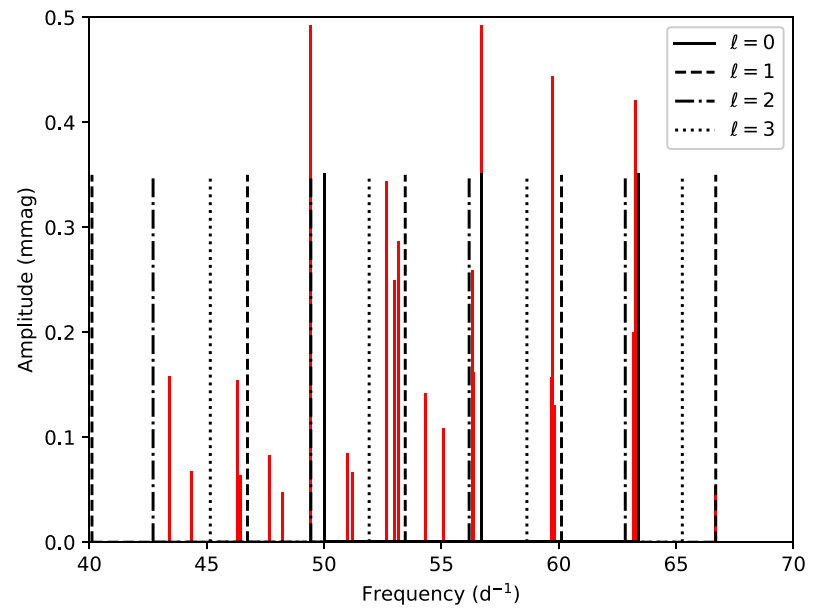

Figure A2. Individual frequency matches for the best-fitting model using the large frequency separation of $\Delta v_{0}=6.707 \mathrm{~d}^{-1}$. The red lines are the observed frequencies plotted versus amplitude, while the black lines are the $\ell=0$ (solid), $\ell=1$ (dashed), $\ell=2$ (dot-dashed), and $\ell=3$ (dotted) model frequencies.

This paper has been typeset from a $\mathrm{T}_{\mathrm{E}} \mathrm{X} / \mathrm{LT} \mathrm{E} \mathrm{X}$ file prepared by the author. 